\title{
Microgravity Environments: The Physical Exercise in the Space
}

Dario Furnari ${ }^{1,2 *}$, Nadya Khan ${ }^{3}$, Melissa Delaney ${ }^{1}$, Margaret Cerna ${ }^{1}$, Khaled Hamlaoui ${ }^{1}$, Sebastien Lagree ${ }^{1,2}$, Amy Peace ${ }^{1,2}$, Megan Owens ${ }^{1,2}$, Shanee Lee Scott ${ }^{1,2}$, Tonja Latham Gustin ${ }^{1,2}$, Tessa Rey ${ }^{1,2}$, Susana Sanchez ${ }^{1}$, Heather Perren ${ }^{1,2}$, Timothy Lee ${ }^{1}$, Michael Cochard ${ }^{1}$, Sandy Wanna ${ }^{1}$, Dayroon Booth ${ }^{1,2}$, Jean N Paul ${ }^{1}$, Jennifer badger ${ }^{1}$, Jim Bermingham ${ }^{1}$, San Soulat ${ }^{1}$

${ }^{1}$ Department of Biomedical Sciences, UK, Germany, Netherlands, USA.

${ }^{2}$ Department of Lagree Studio, USA, Los Angeles.

${ }^{3}$ Department of Pharmacology, College of Medicine and Health Sciences, UAEU.

*Corresponding Author: Dario Furnari, Department of Biomedical Sciences, UK, Germany, Netherlands, USA.

Received Date: 12 June 2021 | Accepted Date: 22 July 2021| Published Date: 02 August 2021

Citation: D Furnari, N Khan, M Delaney, M Cerna, K Hamlaoui. (2021) Microgravity Environments: The Physical Exercise in the Space. Biomedical Research and Clinical Reviews. 4(4); DOI: 10.31579/2692-9406/072

Copyright: (c) 2021 Dario Furnari, This is an open-access article distributed under the terms of the Creative Commons Attribution License, which permits unrestricted use, distribution, and reproduction in any medium, provided the original author and source are credited.

\begin{abstract}
Nice to recede a thesis, transformed into a project and then finally into a great and beautiful research. also the "American magazine Biomedical Science \& Research" publishes an article of mine which is then only an abstract of a work done during my university training where through physical exercise, applied physiology and rehabilitation in particular environments such as microgravity, we can find new methods to improve the quality of life on earth, for example for all those people who suffer from osteoporosis and arthrosis but not only. Exercise is medicine and when administered appropriately it helps to improve people's quality of life. Core, endurance, strength, neuroscience, the secrets for good longevity: heart health, brain health, muscle and joint health. Neuroscience and lagree method, besides fitness and wellness, neuro complex training. Happy to be part of this family and also to be its ambassador and researcher. also used by astronauts returning to earth.
\end{abstract}

Keywords: Microgravity Environments; Physical Exercise; Space

\section{Introduction}

The human body has a great capacity for adaptation, even in the case of significant changes in environmental conditions; like prolonged microgravity.

The force of gravity on the earth produces an acceleration of $1 \mathrm{~g}$ ( $\mathrm{g}$ is the symbol that indicates the acceleration due to gravity). The term microgravity indicates a reduced force of gravity and is therefore used to describe conditions in which the force of gravity is less than that on the earth's surface (less than one g). For example, the gravity force of the moon is only $17 \%$ of that of the earth, or $0.17 \mathrm{~g}$. The term microgravity is often used to describe conditions in space, because the body may not always be in conditions of weightlessness, ie at $0 \mathrm{~g}$.

It is interesting to note that most of the physiological changes due to exposure to microgravity are very similar to those observed in athletes, after a period of inactivity or immobilization, or to changes associated with aging that probably derive from a reduction in physical activity. This similarity is corroborated by the data indicating physical exercise performed during exposure to microgravity as an effective means of counteracting the physiological deterioration that occurs in space. For this reason, but also because exploration in space continues, the influence of microgravity on physical activity is an area of growing interest for sports physiology and exercise specialists.the Space Shuttle spacecraft and on the Orbiting Space Station there is an inversion of the normal posture, that is of the position of the body with respect to the center of gravity, which no longer exists and therefore the mass of blood liquid is redistributed in a different way that on earth. While on Earth gravity retains most of the liquid in the lower limbs, in space, since there is no longer gravity, the liquid collects in the small circle, ie at the level of the pulmonary circulation and at the level of the head. Consequences are water retention in the lung and in the face and brain.

The adaptation mechanisms ensure that everything works the same, but in the photos this phenomenon is clearly seen in the so-called lunar facies or puffy, roundish and tendentially ruddy face. In short, astronauts live in space as a person who lived upside down on Earth.

When they return to Earth, the problems occur at the moment of the transition from microgravity to gravity, that is when they pass from living upside down and suddenly everything turns upside down, at a certain height gravity starts to be felt and astronauts hang from the seat practically they fall on the seat. This step is not gradual because they travel around 27,000 kilometers per hour. The blood from the head flows quickly into the lower limbs and this sudden emptying of blood from the brain causes a short and temporary fall in brain function. It is not a real loss of senses, it does not happen in all the components of the crews but for a few seconds 
someone does not see us anymore and when this has happened to the pilot of the Shuttle, the first few times he has thought. The definitive return to normality however occurs in a couple of days. There is no equivalent disease on Earth that causes the same symptoms. Alterations of the musculoskeletal system (in the space in microgravity, there is loss of muscle mass and loss of bone matrix)

This loss is caused by the lack of bodily statatism, which is an automatic reflex that keeps man on Earth. The main sensors of this reflex are in the soles of the feet and in the legs, therefore, as gravity is lacking, in space it is as if a person was with his feet in the air and therefore the muscles no longer having the stimulus to contraction to maintain the balance, being inactive, they atrophy.

A mechanism of action similar to that which acts on the muscles, is also present in the bones and in microgravity, a hormonal reflex controlled by calcitonin is carried out, so that the bone becomes rarefied, which in technical terms is called osteoporosis.

To counteract muscle reabsorption, astronauts are advised to do a lot of gymnastics, ie cycling, but many do not or do not do much, so when they return to Earth, they feel weak and need to do physical rehabilitation. Bone is a complex structure formed by cells that produce bone, osteoblasts and cells that destroy it, the osteoclasts. These cells are placed on a calcium-based trabecular structure, which is the bone matrix. When the bone matrix is destroyed, it is like rust on a piece of iron, it does not reintegrate anymore.

This condition is common in older people and is called osteoporosis. Spatial osteoporosis affects all astronauts, dissipating 30 to $70 \%$ of the bone, depending on the length of time spent in space. It is an impressive and worrying phenomenon that has affected all Russian astronauts who have remained in space for periods longer than three months.

This is the main current limitation to a space journey to Mars, which otherwise would have already been done; von Braun presented a complete and detailed project, since 1951. The administration of hormonal drugs, such as calcitonin, is currently being studied (2005) to reduce the extent of this disease but for example in the elderly it gives poor results. Because of the fact that in space, the functions of the basic human body are not normal, it is improper to speak of physiology, on the other hand, without talking about pathology, we talk about adaptation.

On the Moon and in the spacecraft, in conditions of absence of protective atmosphere and or in conditions of microgravity, alterations of human bodily functions are produced that are still under study. The known alterations are as follows.

\section{Alterations of the cardiovascular system}

In the Space Shuttle spacecraft and the orbiting space station there is an inversion of the normal clinostatism, ie the position of the body with respect to the center of gravity, which no longer exists and therefore the mass of blood liquid is redistributed in a different way on earth. While on Earth gravity retains most of the fluid in the lower limbs, in space, since there is no longer gravity, the liquid takes place in the small circle, that is at the level of the pulmonary circle and at the level of the head. Consequences are water retention at the pulmonary level and at the level of the face and brain.

The mechanisms of adaptation make sure that everything works the same but in the photos you can clearly see this phenomenon in the so-called lunar facies or face swollen, round and tend to rubizzo. In short, the astronauts live in space as a person who lived on the earth upside down.

When they come back to Earth, the problems happen when they move from microgravity to gravity, that is when they go from living upside down and suddenly everything turns upside down, at a certain height gravity starts to be felt and the astronauts hanging from the seat practically they fall into the seat. This step is not gradual because they travel at speeds of 27,000 kilometers per hour. The blood from the head drains rapidly in the lower limbs and this sudden depletion of blood from the brain causes a brief and temporary fall of brain functions. It is not a real loss of senses, it does not happen in all the crew members but someone for a few seconds does not see us anymore and when this happened to the Shuttle pilot, the first times he gave thought.

The definitive return to normality, however, takes place in a couple of days.There is no equivalent pathology on Earth that causes the same symptom.

\section{Hand Posture Analyzer (HPA)}

In microgravity fatigue has important repercussions on the hands and forearms of the astronauts. Considering that the upper limbs are the main tool of locomotion in space, this can have a significant impact on the performance of astronauts during ordinary work on board the ISS and during extra-vehicular activities. This is one of the reasons why experiments concerning the use of the upper limbs are becoming increasingly important in the context of medical research in space.

Using different scientific protocols it will be possible to determine the degradation of the performance of the musculoskeletal apparatus in microgravity and to favor the studies on the learning mechanisms for motor control.

The results of these experiments will be useful in the development of methods to combat fatigue with the aim of maintaining the condition and improving the performance of the astronauts; this is extremely important from the perspective of future long-term missions. These methods can be used on Earth for the treatment of subjects with local trauma, muscle atrophy, or those suffering from diseases of the Central Nervous System. The Hand Posture Analyzer (HPA), developed by Kaiser Italy as part of the contract with the Italian Space Agency (ASI), was launched on the ISS on the 12P Progress flight in August 2003 and was used during increments 7 and 8 of the ISS by astronauts Ed Lu and Michael Foale.

\section{Antigravity in the study of movement and rehabilitation}

The anti-gravity treadmill produces a lifting force to the patient's body. By lightening the body up to $80 \%$ of its weight, thanks to the variation in pressure inside the air envelope, it allows patients or athletes to walk and run without compromising the biomechanics of movement

It allows patients undergoing hip or knee prostheses to move, walk and run almost without body weight and therefore with less impact, reducing stress on joint joints, neurological patients allows them to regain their mobility in a safe environment, patients trying to lose weight and sports professionals to improve competitive performance.

The human organism has a great capacity for adaptation, even in the case of significant variations in environmental conditions; like prolonged microgravity. The force of gravity on the earth produces an acceleration of $1 \mathrm{~g}$ ( $\mathrm{g}$ is the symbol that indicates the acceleration due to gravity). The term microgravity indicates a reduced gravity force and is therefore used to describe conditions in which the force of gravity is less than that on the earth's surface (less than one g). For example, the gravity of the moon is only $17 \%$ of that of the earth, or $0.17 \mathrm{~g}$. The term microgravity is often used to describe conditions in space, because the body may not always be under weightless conditions, ie $0 \mathrm{~g}$.

It is interesting to note that most of the physiological changes due to exposure to microgravity are very similar to those observed in athletes, after a period of inactivity or immobilization, or changes associated with aging that are likely to result from a reduction in physical activity. This 
similarity is corroborated by data that indicate the physical exercise performed during exposure to microgravity as an effective means of countering the physiological deterioration that occurs in space. For this reason, but also because exploration in space continues, the influence of microgravity on physical activity is an area of increasing interest for specialists in the physiology of sport and exercise (Wilmore-Costill, 2005).

\section{Physiological Modifications Considering Chronic Exposure To Microgravity}

Microgravity endangers the normal functions of the organism. The weight of an object reflects the gravity to which it is subjected and decreases as the object moves away from the earth's surface. At a distance of 12,875 $\mathrm{km}$, for example, body weight is about $25 \%$ of the value on earth. At $337.962 \mathrm{~km}$ the body is weightless because the gravity acceleration is equal to $0 \mathrm{~g}$. In weightlessness, bones that support weight and anti-gravity or postural muscles are weightless. The reduction of the commitment of both the bones and the muscles leads, over time, to their deterioration and compromises their functional capacity. Similar effects have been observed in cardiovascular function.

But what we tend to consider a negative adaptation could represent, in reality, precisely the adaptation necessary to microgravity (WilmoreCostill, 2005).

Astronauts involved in long-duration spaceflight missions are exposed to specific risk factors known to induce profound changes of brain structure and function whose potential long-lasting effects are still under investigation. These changes range from sleep alterations, modifications of brain morphometry, vision impairment, mood shifts, and loss of appetite as well as cognitive deficits, including decrements in attention and executive functions. Among the substantial list of stressors, the effects of microgravity and galactic cosmic radiations constitute the most relevant ones and are at the core of current and future NASA efforts to identify effective countermeasures. Interestingly, while reduced gravity force seems responsible for cephalad fluid shift that potentially affects protein clearance mechanisms, cosmic radiations seem to promote the accumulation of amyloid- $\beta$ in mouse models, induce neuroinflammation, and further alter hippocampal-related cognition. Considering available evidence, a pattern of spaceflight-induced accelerated brain aging seems to emerge in addition to established aging-like effects on cardiovascular and musculoskeletal systems (ie, carotid intima-media thickness increments, inflammatory response, bone loss, muscle atrophy, and DNA telomere modifications, as documented in the recent 1-year long NASA Twin Study). While this raises important issues about astronauts' health, it can also constitute a window into the neurophysiopathology of neurodegenerative processes in humans, which could potentially benefit life on Earth.

\section{Adaptation of bone and tendon to prolonged hindlimb suspension in rats}

The rat hindlimb suspension model was used to ascertain the importance of ground reaction forces in maintaining bone and tendon homeostasis. Young female Sprague-Dawley rats were randomly assigned to either a suspended or a nonsuspended group. After 28 days, femur bones and patellar tendons were obtained for morphological and biochemical analyses. Prolonged suspension induced a significant change in the geometric configuration of the femur middiaphysis by increasing the minimum diameter $(12 \%)$ without any significant alterations in cortical area, density, mineral, and collagen concentrations. Femur wet weight, length, DNA, and uronic acid concentrations of suspended animals were not significantly different from bones of nonsuspended rats. However, the collagen and proteoglycan concentrations in patellar tendons of suspended rats were $28 \%$ lower than the concentrations of matrix proteins in tissues obtained from nonsuspended animals. These data suggest that elimination of ground reaction forces induces alterations in tendon composition and femur diaphyseal shape by changing regional rates in bone remodeling and localized tendon strain. Therefore it appears that ground reaction forces are an important factor in the maintenance of cortical bone and patellar tendon homeostasis during weight-bearing conditions.

\section{References}

1. NASA NCA-1R390-501; Effects of Prolonged Suspension on Dense Fibrous Connective Tissue; Co-Investigator; 1984-1987.

2. NASA NCA2-156; Bone and Tendon Alterations with Prolonged Hindlimb Suspension; CoInvestigator; 1984-1987.

3. Co-Principal Investigator (NASA NCA2-501), Skeletal Effects of Seven Days of Space Flight in Space Lab 3 Rats, 1986-1987.

4. Department of the Navy N66001-87-C0285; Etiology of Tibial Stress Fractures Associated with Strenuous Exercise; CoInvestigator; 1987-1988.

5. Ronald F. Zernicke, PhD, DSc OCTOBER 13, 2020 (M951) 4

6. NASA NAG2-47; Soft Dense Fibrous Connective Tissue Adaptation to Prolonged Suspension; CoPrincipal Investigator; 1987-1988.

7. NASA A53749C; COSMOS Pilot Studies on Ground-Based Control Animal; Co-Principal Investigator.

8. NASA; Connective Tissue Studies in Rats Exposed to 14 Days of Space Flight, COSMOS Mission 1887; Co-Principal Investigator.

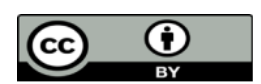

This work is licensed under Creative Commons Attribution 4.0 License

To Submit Your Article Click Here: Submit Manuscript

DOI: $10.31579 / 2692-9406 / 072$

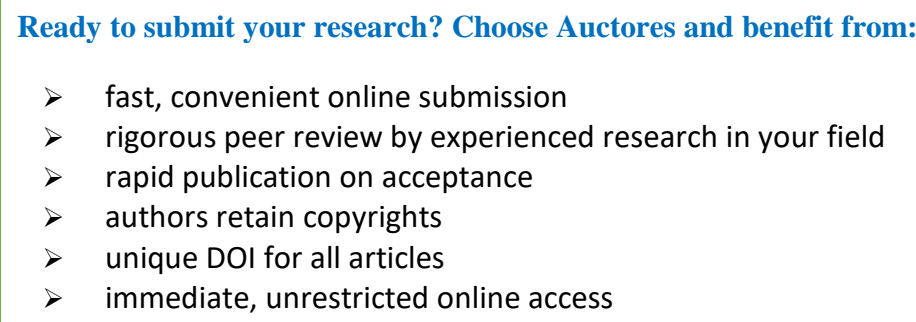

Ready to submit your research? Choose Auctores and benefit from:

> fast, convenient online submission

$>\quad$ rigorous peer review by experienced research in your field

$>$ rapid publication on acceptance

$>$ authors retain copyrights

$>$ unique DOI for all articles

$>$ immediate, unrestricted online access

At Auctores, research is always in progress.

Learn more www.auctoresonline.org/journals/dermatology-anddermatitiss- 